\title{
Interval Development of Multiple Sub-Segmental Pulmonary Embolism in Mycoplasma Pneumoniae Bronchiolitis and Pneumonia
}

\author{
Peter V. Bui ${ }^{1}$ \\ Sapna Bhatia ${ }^{2}$ \\ Ali I. Saeed ${ }^{2}$ \\ ${ }^{1}$ Department of Internal Medicine \\ ${ }^{2}$ Division of Pulmonary, Critical Care, and Sleep Medicine \\ The University of New Mexico \\ Albuquerque, NM, USA
}

\begin{abstract}
Introduction: Cases of pulmonary embolism (PE) concurrent with Mycoplasma pneumoniae infection are rare in the medical literature. We describe a patient with $M$. pneumoniae bronchiolitis and pneumonia who developed multiple right-sided, subsegmental PE.

Case Description: A 54-year-old man presented following one week of respiratory and constitutional symptoms. He was admitted for respiratory distress and started on ceftriaxone, azithromycin, and oseltamivir. Because of a lack of clinical improvement, antibiotics were escalated to vancomycin and piperacillin-tazobactam. M. pneumoniae $\operatorname{lgM}$ and IgG serologies returned positive, and antibiotics were narrowed to azithromycin, with clinical improvement and gradual decrease in supplemental oxygen requirement. One week into the hospitalization, the patient abruptly developed an increased oxygen requirement. Computed tomography angiography (CTA) of the chest found stable M. pneumoniae bronchiolitis and pneumonia and the interval development of multiple right-sided, sub-segmental PE. He was treated with unfractionated and then low-molecular-weight heparin as a bridge to warfarin, azithromycin, and a prednisone taper. In the outpatient setting, repeat CTA revealed resolution of $M$. pneumoniae infection and PE.

Discussion: Although the mechanism and association are unclear, other case reports have proposed that $M$. pneumoniae infection promotes hypercoagulability or a prothrombotic state, predisposing patients to thromboembolism. In a patient with $M$. pneumoniae infection who develops sudden respiratory distress or failure despite appropriate treatment, clinicians should have a high suspicion for PE, and a CTA should be considered as part of further evaluation.
\end{abstract}

\section{Introduction}

Mycoplasma pneumoniae is one of thirteen Mycoplasma species isolated from humans and less commonly causes lower respiratory tract infections, of which atypical pneumonia occurs at higher rates (1). These lower respiratory tract infections have been reported to present similarly to other disease processes such as asthma and pulmonary embolism (PE) $(2,3)$. M. pneumoniae pneumonia typically has a benign course with low mortality. A study by von Baum et al. found a mortality of $0.7 \%$ in 
patients with $M$. pneumoniae pneumonia, with the deaths occurring in hospitalized patients (4). Despite this low mortality, rare complications may contribute to morbidity and mortality, although to what degree, if any, is unclear. A case report in the medical literature describes a PE and a hypercoagulable state associated with $M$. pneumoniae pneumonia in an adult during the peri-infectious period (5). We present a case with radiographic evidence of the interval development of multiple segmental PE in a patient with M. pneumoniae bronchiolitis and pneumonia.

\section{Case Description}

A 54-year-old man with a 15-pack-year smoking history, positive purified protein derivative treated with isoniazid, occupational exposures including asbestos and dust, and a current history of ethanol abuse presented to the emergency department with a one-week history of a productive cough with yellow sputum, weakness, shortness of breath, and dyspnea on exertion. He also noticed diffuse papular cutaneous lesions over his back.

In the emergency department, he was hypoxic with a need for supplemental oxygen. Cardiopulmonary examination was unremarkable. Initial laboratory studies including complete blood count, chemistry panel, and hepatic function panel were notable for a leukocytosis of $13.6 \mathrm{k} / \mathrm{\mu L}$ with a neutrophilia of $83 \%$, aspartate transaminase of 108 units/L, alanine transaminase of 152 units/L, alkaline phosphatase of 175 units/L, and total bilirubin of $1.5 \mathrm{mg} / \mathrm{dL}$, and creatine kinase of 563 units/L. Conventional chest radiograph (Figure 1) showed a left lower lobe infiltrate.
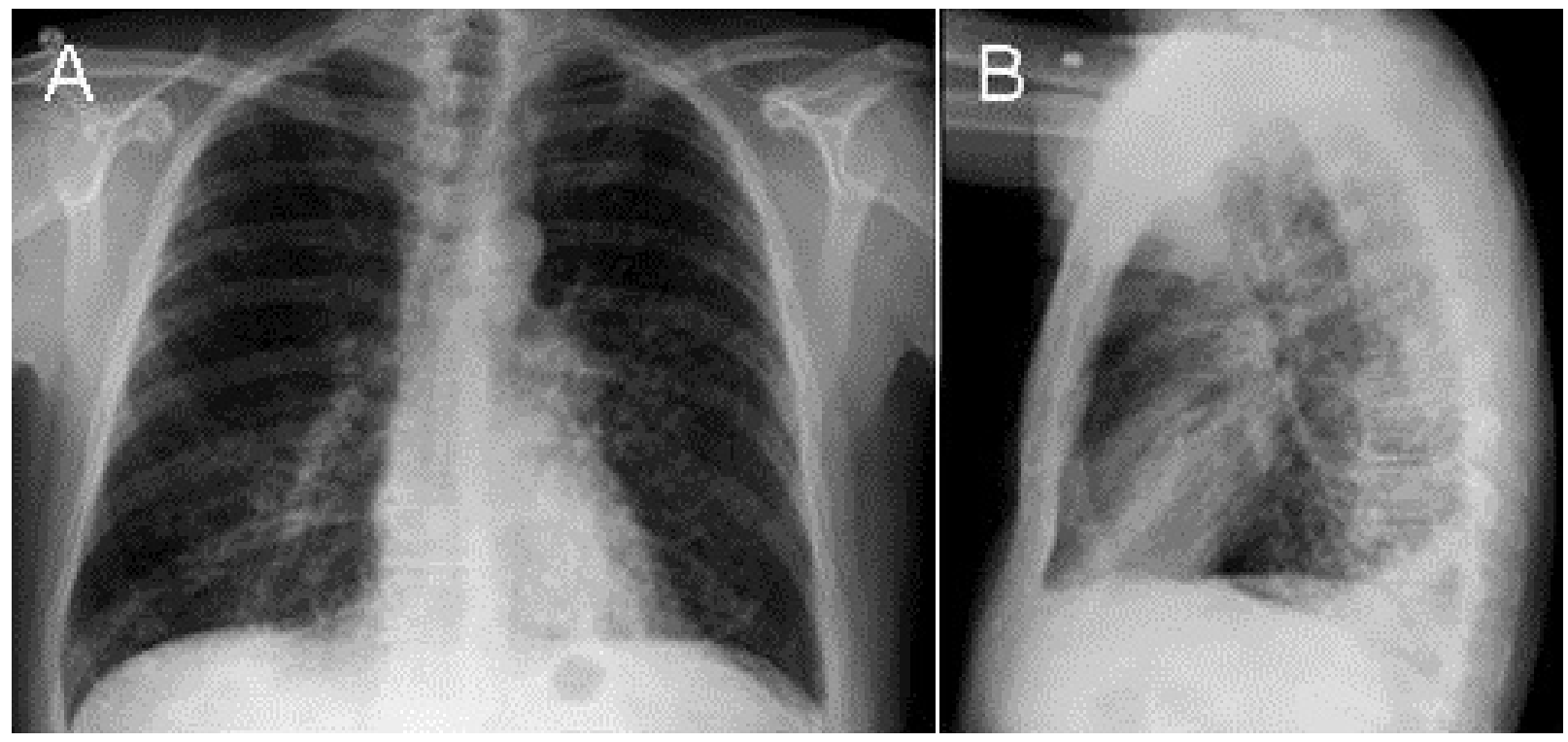

Figure 1. Conventional chest radiograph on day zero of the hospitalization. The images show a left lower lobe infiltrate. 
The patient was admitted to the hospital and started on ceftriaxone and azithromycin for community-acquired pneumonia as well as oseltamivir over concerns for influenza.

During the initial hospitalization, the patient required supplemental oxygen for hypoxia with a rapid increase in fractional inspired oxygen $\left(\mathrm{F}_{i} \mathrm{O}_{2}\right)$ to maintain oxygen saturation above $90 \%$. Because of a lack of clinical improvement, antibiotics were broadened to include vancomycin and piperacillin-tazobactam. Since he continued to require a $\mathrm{F}_{i} \mathrm{O}_{2}$ of $60 \%$ on day four of the hospitalization, additional workup for atypical bacterial, viral, and fungal pathogens were performed after consultation with pulmonology. Acid-fast bacillus cultures and stains were negative. Sputum cultures were not obtained. An arterial blood gas prior to evaluation by Pulmonology found a $\mathrm{pH}$ of 7.42 , partial pressure of carbon dioxide of $38 \mathrm{mmHg}$, partial pressure of oxygen of $86 \mathrm{mmHg}, \mathrm{HCO}_{3}$ of $24 \mathrm{mmol} / \mathrm{L}$, and $\mathrm{F}_{\mathrm{i}} \mathrm{O}_{2}$ of $95 \%$. Computed tomography (CT) of the chest (Figure 2) showed extensive bronchiolitis with focal areas of consolidation involving bilateral lower lobes.

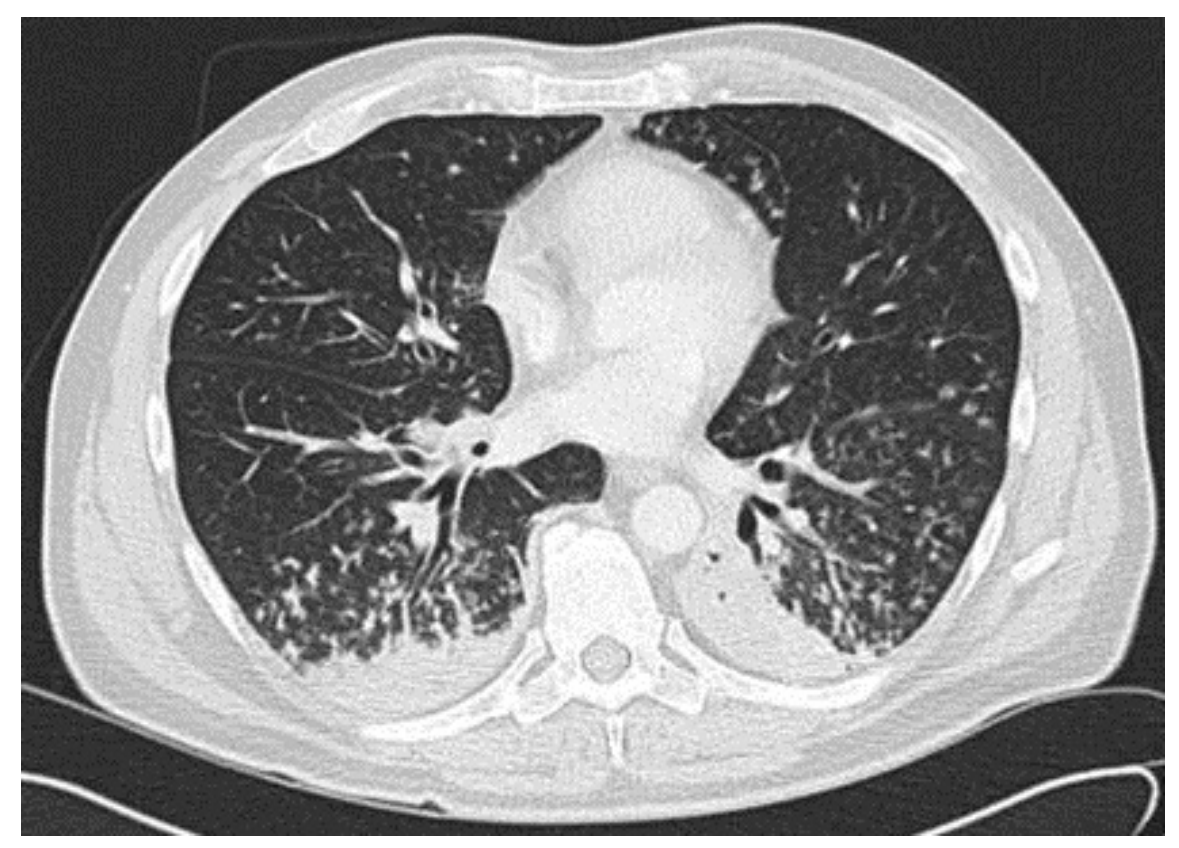

Figure 2. Computed tomography of the chest on day four of the hospitalization. The image shows an extensive bronchiolitis with focal areas of consolidation involving bilateral lower lobes.

Oseltamivir was discontinued after the respiratory viral panel returned negative. Broad spectrum antibiotics were narrowed to azithromycin after M. pneumoniae IgM and IgG serologies returned positive. His oxygen requirement gradually improved over the next two days, and he was transitioned to nasal cannula.

On day seven of his hospitalization, the patient suddenly developed moderate respiratory distress with an increase in oxygen requirement. CT angiography (CTA) of 
the chest (Figure 3 ) done at this juncture showed unchanged parenchymal findings with interval development of multiple sub-segmental pulmonary emboli in the right lung.

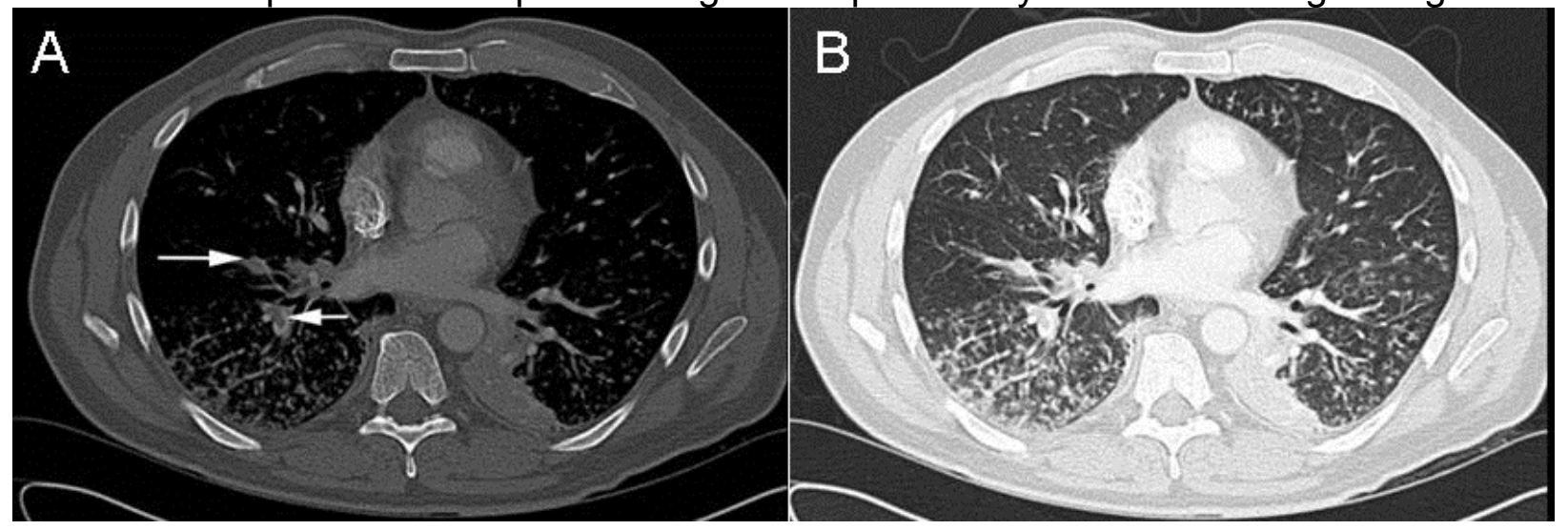

Figure 3. Computed tomography angiography of the chest on day five of the hospitalization. The images show unchanged parenchymal findings with interval development of multiple sub-segmental pulmonary emboli in the right lung (see white arrows in Figure $3 \mathrm{~A}$ ).

Doppler ultrasound found no evidence of deep venous thrombosis (DVT) in both lower extremities. He was subsequently started on therapeutic anticoagulation with unfractionated heparin and then low-molecular-weight heparin as a bridge to warfarin. The patient subsequently improved on a 14-day course of azithromycin $500 \mathrm{mg}$ orally once daily and 3-month tapered course of prednisone $60 \mathrm{mg}$ orally once daily for $M$. pneumoniae infection, a 3-month course of warfarin for the PE, and supplemental oxygen. During follow-up in the outpatient setting, CTA of the chest showed the infection and PE to have resolved, and all therapies related to the infection and PE were discontinued.

\section{Discussion}

We herein describe a case of $M$. pneumoniae bronchiolitis and pneumonia complicated by right-sided PE. The reported occurrences of venous thromboembolism (VTE) during $M$. pneumoniae infection are limited to case reports. In our review of the literature, we found one case of $M$. pneumoniae infection associated with PE in the adult population. Ascer et al. (5) presented the case of a 28-year-old male with right-sided pneumonia and right-sided PE who was found to have antiphospholipid antibodies. For the PE, this patient was successfully treated with recombinant tissue-type and plasminogen activator and heparin and was discharged with hydroxychloroquine sulfate, aspirin, and warfarin. However, Ascer did not publish additional follow up for this seemingly prothrombotic state. In a case without PE, Senda et al. (6) reported on a 21-year-old patient with a left middle cerebral artery embolus and DVT in bilateral femoral veins in the setting of a M. pneumoniae infection. This patient had a transient increase in prothrombin time, partial thromboplastin time, fibrin/fibrinogen degradation products, thrombin-antithrombin III-complex, antiphospholipid antibodies, and IgM anticardiolipin antibodies and decrease in protein $\mathrm{C}$ activity. 
The pediatric medical literature has additional case reports linking $M$. pneumoniae to PE. Brown et al. (7) described a 6-year-old male child with $M$. pneumoniae pneumonia, right-sided ileofemoral thrombosis, and right-sided PE found to have anticardiolipin IgG and IgM antibodies, lupus anticoagulant, and acquired activated protein $\mathrm{C}$ resistance. This prothrombotic state subsequently resolved after treatment of the infection with antibiotics and the PE with unfractionated heparin and then dalteparin. In another case report, during workup for a 13-year-old male child with right-sided PE in the setting of a left lower lobe M. pneumoniae pneumonia, Graw-Panzer et al. (8) found lupus anticoagulant, anticardiolipin $\lg G$ and $\lg M$ antibodies, and an underlying protein $S$ deficiency. The transient prothrombotic markers returned to normal levels during subsequent follow-up for his acute illness.

M. pneumoniae pulmonary infections have been reported in the pediatric medical literature to be associated with an underlying hypercoagulability. Creagh et al. (9) reported on a left femoral vein thrombosis in a 10-year-old female with $M$. pneumoniae pneumonia who was found to have type I familial antithrombin III deficiency. In another case report of two children describing splenic infarcts associated with $M$. pneumoniae pneumonia, Witmer et al. (10) found elevated D-dimer, lupus anticoagulant, and elevated anticardiolipin and $\beta 2$-glycoprotein antibodies that resolved following successful treatment of the infection with antibiotics and a three-month course of anticoagulation and, in one patient, an additional course of aspirin (10). No specific etiology was found for the infarctions, but Witmer et al. attributed the infarctions to possible thrombosis. Other case reports in the pediatric literature that found antiphospholipid antibodies include a patient with cardiac thrombus and internal carotid artery occlusion $(11,12)$. However, in their report of right popliteal artery thrombosis in a 5 -year-old male child with M. pneumoniae pneumonia and right popliteal artery thrombosis, Joo et al. (13) did not find abnormalities in their limited hypercoagulability workup.

Our lack of hypercoagulability workup limits comparison with the available medical literature. We did not perform a hypercoagulability workup because the patient did not meet any Wells criteria and did not have a family history of hypercoagulability. Based on the available case reports, the underlying pathophysiology can be inferred to be related to a transient formation of antiphospholipid antibodies during a M. pneumoniae infection. Additionally, the thromboembolism can be expected to occur within a short period of time following the onset of symptoms. The rate that hypercoagulability occurs in infected patients and the practical clinical relevance of such a prothrombotic state without or without an inherited or congenital deficiency are unknown at this time. These questions would benefit from further investigation.

An alternative interpretation is a preexisting hypercoagulability may predispose patients to $M$. pneumoniae infection, which can exacerbate the hypercoagulability, further increasing the risk of VTE. This interpretation may be relevant for the patients of GrawPanzer et al. (8) and Creagh et al. (9) who had underlying hypercoagulable conditions and subsequently suffered $M$. pneumoniae infection and then developed VTE. The 
Worcester Venous Thromboembolism study found an association between infection and VTE, and Rosendaal's review of the literature found an association between hypercoagulability and increased risk of thrombosis (14-16). With the available case reports and epidemiological studies, this alternative interpretation has not been elucidated.

In this report, we described the interval development of PE in a patient with $M$. pnuemoniae bronchiolitis and pneumonia. The mechanism for the hypercoagulability during M. pneumoniae infection is unclear. A CTA of the chest should be obtained if a patient with $\mathrm{M}$. pneumonia infection fails to show clinical improvement or suddenly develops clinical worsening of his or her respiratory status, so as to exclude PE. However, clinicians should take into account that Mycoplasma pneumonia may present with the symptoms of PE (3).

\section{Acknowledgements}

We would like to acknowledge Cecelia Kieu for assisting in the preparation of the figures for this manuscript.

\section{References}

1. Cha SI, Shin KM, Kim M, Yoon WK, Lee SY, Kim CH, Park JY, Jung TH. Mycoplasma pneumoniae bronchiolitis in adults: Clinicoradiologic features and clinical course. Scand J Infect Dis. 2009;41(6-7):515-9. [CrossRef] [PubMed]

2. Vasudevan VP, Suryanarayanan M, Shahzad S, Megjhani M. Mycoplasma pneumonia bronchiolitis mimicking asthma in an adult. Respir Care. 2012;57(11):1974-6. [CrossRef] [PubMed]

3. Simmons BP, Aber RC. Mycoplasma pneumoniae pneumonia. Symptoms mimicking pulmonary embolism with infarction. JAMA. 1979;241(12):1268-9. [CrossRef] [PubMed]

4. von Baum H, Welte T, Marre R, Suttorp N, Luck C, Ewig S. Mycoplasma pneumoniae pneumonia revisited within the German Competence Network for Community-acquired pneumonia (CAPNETZ). BMC Infect Dis. 2009;9;62. [CrossRef] [PubMed]

5. Ascer E, Marques M, Gidlund M. M pneumonia infection, pulmonary thromboembolism and antiphospholipid antibodies. BMJ Case Rep. 2011;2011. [CrossRef] [PubMed]

6. Senda J, Ito M, Atsuta N, Watanabe H, Hattori N, Kawai H, Sobue g. Paradoxical brain embolism induced by Mycoplasma pneumoniae infection with deep venous thrombosis. Intern Med. 2010;49(18):2003-5. [CrossRef] [PubMed]

7. Brown SM, Padley S, Bush A, Cummins D, Davidson S, Buchdahl R. Mycoplasma pneumonia and pulmonary embolism in a child due to acquired prothrombotic factors. Pediatr Pulmonolo. 2008;43(2):200-202. [CrossRef] [PubMed]

8. Graw-Panzer KD, Verma S, Rao S, Miller ST, Lee H. Venous thrombosis and pulmonary embolism in a child with pneumonia due to Mycoplasma pneumoniae. $J$ Natl Med Assoc. 2009;101(9):956-8. [PubMed] 
9. Creagh MD, Roberts IF, Clark DJ, Preston FE. Familial antithrombin III deficiency and Mycoplasma pneumoniae pneumonia. J Clin Pathol. 1991;44:870-1. [CrossRef] [PubMed]

10. Witmer CM, Steenhoff AP, Shah SS, Raffini LJ. Mycoplasma pneumoniae, splenic infarct, and transient antiphospholipid antibodies: a new association? Pediatrics. 2007;119:292-5. [CrossRef] [PubMed]

11. Bakshi M, Khemani C, Vishwanathan V, Anand RK. Mycoplasma pneumonia with antiphospholipid antibodies and a cardiac thrombus. Lupus 2006;15:105-6. [CrossRef] [PubMed]

12. Tanir G, Aydemir C, Yilmaz D, Tuygun N. Internal carotid artery occlusion associated with Mycoplasma pneumoniae infection in a child. Turk J Pediatr. 2006;48(2):166-71. [PubMed]

13. Joo CU, Kim JS, Han YM. Mycoplasma pneumoniae induced popliteal artery thrombosis treated with urokinase. Postgrad Med J. 2001;77:723-724. [CrossRef] [PubMed]

14. Rosendaal FR. Venous thrombosis: a multicausal disease. Lancet. 1999;353(9159):1167-73. [PubMed]

15. Spencer FA, Emery C, Joffe SW, Pacifico L, Lessard D, Reed G, Gore JM, Goldberg RJ. Incidence rates, clinical profile, and outcomes of patients with venous thromboembolism. The Worcester VTE study. J Thromb Thrombolysis. 2009;28(4):401-9. [CrossRef] [PubMed]

16. Spencer FA, Emery C, Lessard D, Anderson F, Emani S, Aragam J, Becker RC, Goldberg RJ. The Worcester Venous Thromboembolism study: a population-based study of the clinical epidemiology of venous thromboembolism. J Gen Intern Med. 2006;21(7):722-7. [CrossRef] [PubMed] 